\title{
New Application of Functional Integrals to Classical Mechanics
}

\author{
Anton Zherebtsov ${ }^{\dagger \star}$, Kirill Ilinski ${ }^{\star}$ \\ ${ }^{\dagger}$ Division of Computational Physics, Department of Physics, St.Petersburg \\ State University, St.Petersburg 198904, Russia. \\ e-mail: anton.zherebtsov@fusionam.com \\ ^Fusion Asset Management, 23 Berkeley Square, London W1J6 HE, UK. \\ e-mail: kirill.ilinski@fusionam.com
}

\begin{abstract}
In this paper a new functional integral representation for classical dynamics is introduced. It is achieved by rewriting the Liouville picture in terms of bosonic creation-annihilation operators and utilizing the standard derivation of functional integrals for dynamical quantities in the coherent states representation. This results in a new class of functional integrals which are exactly solvable and can be found explicitly when the underlying classical systems are integrable.
\end{abstract}

Keywords: Classical mechanics, functional integrals.

\section{Introduction}

Functional integrals are very popular in quantum mechanics and quantum statistical physics as a basic tool of both perturbative and non-perturbative analysis. The most fruitful applications of the functional integrals include Faddeev-Popov ghosts in the field theory [1], non-perturbative corrections to the standard perturbation theory series [2] 3], functional formalism for disordered systems 4], introduction of collective variables based on Stratonovich-Hubbard transformation(see [5] and refs therein) and applications to algebraic and differential geometry [6, 7, 8].

The beauty of the functional integrals lies in the fact that they essentially reduce quantum mechanical problems to a classical mechanical picture with an 
additional stochastic noise. This means that instead of necessity of dealing with operators and partial differential equations (sometimes infinitely dimensional) one can think about the problem in terms of classical observables and, at the final stage, to average them across possible trajectories generated by (quantum) noise. This observation allows to find a formal expression for any quantum observable and is a cornerstone of all mentioned above applications.

If functional integrals bring quantum mechanics back to classics, it is possible to formulate classical dynamics in terms of quantum one and therefore benefit from a quantum-mechanical toolbox. The question is not new. In last ten years series of papers 9] examined one of possible ways to introduce the path integral formalism into the classical mechanics. Although bringing about interesting mathematics they, in our view, did not result in a workable quantum-mechanical formalism which would allow a user to employ many useful functional-integral tricks which proved to be so helpful in the theory of many interacting degrees of freedom, disordered systems and renormalisation group. In this paper we are trying to fill the gap and to introduce a functional integral representation of classical dynamics which is similar in many respects to the standard functional integrals of many-body theory and allows perturbative and non-perturbative treatment of classical many-body systems and classical random dynamics. As a by-product we also obtain class of quantum mechanical systems which are related in our formalism to classical mechanics and therefore allow exact solutions.

The paper is organized as follows. In the next section we briefly remind the Liouville formulation of classical dynamics, outline the main idea of the paper and introduce the creation-annihilation pairs for classical momentum and coordinates. Section 2 treats in great detail the case of one-dimensional classical harmonic oscillator. It is shown that there exists a two-dimensional quadratic quantum mechanical system which is equivalent to the classical oscillator in the sense that all quantum dynamical charatecteristics can be expressed through the corresponding classical trajectories. Section 3 builds on this and describes a general n-dimensional construction in the case of arbitrary classical potential. This results in the description of a new class of functional integrals and the corresponding quantum systems which are exactly solvable and which dynamical quantities can be found explicitly if the underlying classical systems are integrable. Section 4 concludes the paper with a list of possible application of the developed formalism. 


\section{Classical Mechanics in the coherent represen- tation}

Let us consider a mechanical system with the phase space $\mathcal{M}=\{p, q\}^{1}$, algebra of observables $\mathcal{A}$, set of states $\mathcal{S}$, and Hamiltonian $H$. Assume that the phase space $\mathcal{M}$ of the mechanical system has a volume form invariant under the phase flow with Hamiltonian $H$. Denoting this volume form by $d x$, we can write a probability measure $d \mu$ as $d \mu(x)=\rho(x, t) d x$, where $\rho(x, t)$ is a positive distribution (generalized function) on $\mathcal{M}$. For the pure state supported at $x_{0} \in \mathcal{M}$ we have $\rho(x, t)=\delta\left(x-x_{0}\right)$ Dirac $\delta$-function. This representation introduces Liouvilles picture, in which observables do not depend on time

$$
\frac{d f}{d t}=0, \quad f \in \mathcal{A},
$$

and states $d \mu(x)=\rho(x, t) d x$ satisfy Liouvilles equation,

$$
\frac{\partial \rho(x, t)}{\partial t}=-\{H, \rho\}, \quad \rho(x, t) d x \in \mathcal{S},
$$

which is understood in the distributional sense, and Poisson brackets is given by:

$$
\{f, g\}=\frac{\partial f}{\partial p} \frac{\partial g}{\partial q}-\frac{\partial f}{\partial q} \frac{\partial g}{\partial p} .
$$

We can rewrite this equation in Schrödinger manner form:

$$
i \frac{\partial \rho(p, q ; t)}{\partial t}=\mathcal{L}(p, q) \rho(p, q ; t) .
$$

where $\mathcal{L}$ is Liouville operator determined as

$$
\mathcal{L}(p, q)=-i \frac{\partial H}{\partial p} \frac{\partial}{\partial q}+i \frac{\partial H}{\partial q} \frac{\partial}{\partial p} .
$$

Every solution of the initial value problem: $\left.\rho(p, q ; t)\right|_{t=0}=\rho\left(p^{\prime}, q^{\prime}, 0\right)$ for the Equation (11) can be written in terms of the evolution operator $U(t)=$ $e^{-i t \mathcal{L}}$, which is a unitary operator that relates the state functions of the system $\rho(p, q ; T)$ with $\rho\left(p^{\prime}, q^{\prime} ; 0\right)$. Operator $U(t)$ is an integral operator with the kernel $\mathcal{U}\left(p, q \mid p^{\prime} q^{\prime}\right)$ and we can write:

$$
\rho(p, q)=\int \mathcal{U}\left(p, q \mid p^{\prime}, q^{\prime}\right) \rho\left(p^{\prime}, q^{\prime}\right) d p^{\prime} d q^{\prime}
$$

\footnotetext{
${ }^{1}$ To keep formulae simple in this section we do not stress that both $p$ and $q$ can be ndimensional vectors.
} 

$a_{2}^{\dagger}$ :

The next step is to introduce creation and annihilation operators $a_{1}, a_{2}, a_{1}^{\dagger}$,

$$
\begin{array}{ll}
a_{1}=\frac{1}{\sqrt{2}}\left(p+\frac{\partial}{\partial p}\right), & a_{1}^{\dagger}=\frac{1}{\sqrt{2}}\left(p-\frac{\partial}{\partial p}\right), \\
a_{2}=\frac{1}{\sqrt{2}}\left(q+\frac{\partial}{\partial q}\right), & a_{2}^{\dagger}=\frac{1}{\sqrt{2}}\left(q-\frac{\partial}{\partial q}\right)
\end{array}
$$

with standard commutation relations

$$
\left[a_{1}, a_{1}^{\dagger}\right]=1, \quad\left[a_{2}, a_{2}^{\dagger}\right]=1,
$$

all other commutators are 0 . These operators allow us to define the corresponding coherent states which are eigenstates of the operators $a=\left(a_{1}, a_{2}\right)$, $a^{\dagger}=\left(a_{1}^{\dagger}, a_{2}^{\dagger}\right)$ :

$$
\begin{gathered}
|z\rangle=\left|z_{1} z_{2}\right\rangle=\sum_{n=0}^{\infty} \frac{\left(a_{1}^{\dagger} z_{1}\right)^{n}}{n !} \sum_{m=0}^{\infty} \frac{\left(a_{2}^{\dagger} z_{2}\right)^{m}}{m !}|0\rangle|0\rangle e^{-\frac{1}{2}\left|z_{1}\right|^{2}} e^{-\frac{1}{2}\left|z_{2}\right|^{2}} \\
\langle\bar{z}|=\left\langle\bar{z}_{1} \bar{z}_{2}\right|=\langle 0|\langle 0| \sum_{n=0}^{\infty} \frac{\left(a_{1} \bar{z}_{1}\right)^{n}}{n !} \sum_{m=0}^{\infty} \frac{\left(a_{2} \bar{z}_{2}\right)^{m}}{m !} e^{-\frac{1}{2}\left|\bar{z}_{1}\right|^{2}} e^{-\frac{1}{2}\left|\bar{z}_{2}\right|^{2}} .
\end{gathered}
$$

Here $|0\rangle$ is defined by the equation $a|0\rangle=0$ and unit decomposition is:

$$
I=\int \frac{d z d \bar{z}}{2 \pi i}|z\rangle\langle\bar{z}|
$$

It is not difficult to find the coherent states in the coordinate representation by multiplying this equation from the left with $\langle x|$ we now get $\langle x|a| 0\rangle=0$, or

$$
\left\langle x\left|\frac{1}{\sqrt{2}}\left(x+\frac{\partial}{\partial x}\right)\right| 0\right\rangle=0 .
$$

This differential equation is simple to solve, and we obtain

$$
\langle x \mid 0\rangle=\left(\frac{1}{\pi}\right)^{1 / 4} e^{-x^{2} / 2} .
$$

In similar manner one can find the $x$-representation of other eigenstates $|n\rangle$ by applying the operator $\left(a^{\dagger}\right)^{n}$ to the ground state:

$$
\langle x \mid n\rangle=\left\langle x\left|\frac{1}{\sqrt{n !}}\left(a^{\dagger}\right)^{n}\right| 0\right\rangle=\frac{H_{n}(x)}{\sqrt{2^{n} n !} \sqrt[4]{\pi}} e^{-\frac{x^{2}}{2}} .
$$

Here $H_{n}(x)=e^{x^{2} / 2}\left(x-\frac{d}{d x}\right)^{n} e^{-x^{2} / 2}$ is the $n$-th Hermite polynomial. It is now straitforward to write down the coherent states in $x$-representation:

$$
\begin{aligned}
\langle x \mid z\rangle & =\left\langle x\left|e^{-\frac{|z|^{2}}{2}} \sum_{n=0}^{\infty} \frac{z^{n}}{\sqrt{n !}}\right| n\right\rangle=e^{-\frac{|z|^{2}}{2}} \sum_{n=0}^{\infty} \frac{z^{n} H_{n}(x)}{n ! \sqrt[4]{\pi} \sqrt{2^{n}}} e^{-\frac{x^{2}}{2}} \\
& =\frac{1}{\sqrt[4]{\pi}} e^{-\frac{|z|^{2}}{2}-\frac{x^{2}}{2}} \sum_{n=0}^{\infty} \frac{(z / \sqrt{2})^{n}}{n !} H_{n}(x)=\frac{1}{\sqrt[4]{\pi}} e^{-\frac{|z|^{2}}{2}-\frac{x^{2}}{2}} e^{-\frac{z^{2}}{2}+\sqrt{2} x z}(6)
\end{aligned}
$$


where we used well known formula for the generating function of Hermite polynomials: $\sum_{n=0}^{\infty} H_{n}(x) \frac{s^{n}}{n !}=\exp \left\{-s^{2}+2 s x\right\}$. The same analysis yields the following expression for the conjugated coherent states:

$$
\langle\bar{z} \mid x\rangle=\frac{1}{\sqrt[4]{\pi}} \exp \left\{-\frac{|\bar{z}|^{2}}{2}-\frac{x^{2}}{2}-\frac{\bar{z}^{2}}{2}+\sqrt{2} x \bar{z}\right\}
$$

The coherent states are the basis for derivation of the functional integral. As soon as the Liouville operator (2) is expresses in terms the creation-annihilation operators $a_{1,2}, a_{1,2}^{\dagger}$ in the so-called Normal form (i.e. when all creation operators stand to the left of the annihilation operators $), \mathcal{L}_{\text {norm }}\left(a^{\dagger}, a\right)$, one can find the evolution operator $U\left(p, q \mid p^{\prime}, q^{\prime}\right)$ as

$$
\begin{aligned}
U\left(p, q \mid p^{\prime}, q^{\prime}\right) & =\left\langle x|\mathcal{U}(T, 0)| x^{\prime}\right\rangle \\
& =\int d z d \bar{z} d z^{\prime} d \bar{z}^{\prime}\langle x \mid z\rangle\left\langle\bar{z}|\mathcal{U}(T, 0)| z^{\prime}\right\rangle\left\langle\bar{z}^{\prime} \mid x^{\prime}\right\rangle,
\end{aligned}
$$

where $d z d \bar{z}=\frac{d z_{1} d \bar{z}_{1}}{2 \pi i} \frac{d z_{2} d \bar{z}_{2}}{2 \pi i}$ and $d z^{\prime} d \bar{z}^{\prime}=\frac{d z_{1}^{\prime} d \bar{z}_{1}^{\prime}}{2 \pi i} \frac{d z_{2}^{\prime} d \bar{z}_{1}^{\prime}}{2 \pi i}$ and the kernel of evolution operator is given by the following functional integral [5]:

$$
\begin{aligned}
& \left\langle\bar{z}_{1} \bar{z}_{2}|\mathcal{U}(T, 0)| z_{1}^{\prime} z_{2}^{\prime}\right\rangle=\int \mathcal{D} \psi_{1} \mathcal{D} \bar{\psi}_{1} \mathcal{D} \bar{\psi}_{2} \mathcal{D} \psi_{2} \\
& \exp \left\{\sum_{k=1}^{2} \bar{\psi}_{k}(T) \psi_{k}(T)-\frac{1}{2}\left|\bar{\psi}_{k}(T)\right|^{2}-\frac{1}{2}\left|\psi_{k}(0)\right|^{2}\right. \\
& \left.+\int_{0}^{T}\left(-\bar{\psi}_{1}(t) \dot{\psi}_{1}(t)-\bar{\psi}_{2}(t) \dot{\psi}_{2}(t)-i \mathcal{L}_{n o r m}\left(\bar{\psi}_{1}, \bar{\psi}_{2}, \psi_{1}, \psi_{2}\right)\right) d t\right\} .
\end{aligned}
$$

Here $\mathcal{D} \bar{\psi}_{1} \mathcal{D} \psi_{1}=\prod_{t} \frac{d \bar{\psi}_{1} d \psi_{1}}{2 \pi i}, \mathcal{D} \bar{\psi}_{2} \mathcal{D} \psi_{2}=\prod_{t} \frac{d \bar{\psi}_{2} d \psi_{2}}{2 \pi i}$ and initial conditions are:

$$
\begin{array}{cc}
\psi_{1}(0)=z_{1}^{\prime}, & \psi_{2}(0)=z_{2}^{\prime} \\
\bar{\psi}_{1}(T)=\overline{z_{1}}, & \bar{\psi}_{2}(T)=\overline{z_{2}}
\end{array}
$$

This is the functional integral representation of the classical dynamics we study in the paper.

\section{Harmonic oscillator}

Before moving further, we would like to consider a simple example which became rather customary to test new techniques, the harmonic oscillator. In this section we apply the derived above functional integral representation for the evolution 
operator of classical harmonic oscillator with hamiltonian $H(p, q)=\frac{\omega}{2}\left(p^{2}+q^{2}\right)$. The kernel of the classical evolution operator or propagator gives us solutions of the classical dynamical equations:

$$
\dot{p}=-\frac{\partial H}{\partial q}, \quad \dot{q}=\frac{\partial H}{\partial p} .
$$

or

$$
U\left(q, p \mid q^{\prime}, p^{\prime}\right)=\left\langle q, p \mid q^{\prime}, p^{\prime}\right\rangle=\delta\left(q-\widetilde{q}^{\prime}\right) \delta\left(p-\widetilde{p}^{\prime}\right), \quad T \geq 0
$$

with

$$
\widetilde{p}^{\prime}=p^{\prime} \cos \omega T-q^{\prime} \sin \omega T, \quad \widetilde{q}^{\prime}=q^{\prime} \cos \omega T+p^{\prime} \sin \omega T .
$$

and thus completely describes system behavior. We now obtain these expressions from the functional integral for the evolution operator.

Using Equation (2) one can derive the following expression for the Liouville operator:

$$
\mathcal{L}(p, q)=i \omega\left(q \frac{\partial}{\partial p}-p \frac{\partial}{\partial q}\right),
$$

which can be easily expressed in the terms creation and annihilation operators $a_{1}, a_{2}, a_{1}^{\dagger}, a_{2}^{\dagger}$ :

$$
\begin{array}{ll}
a_{1}=\frac{1}{\sqrt{2}}\left(p+\frac{\partial}{\partial p}\right), & a_{1}^{\dagger}=\frac{1}{\sqrt{2}}\left(p-\frac{\partial}{\partial p}\right), \\
a_{2}=\frac{1}{\sqrt{2}}\left(q+\frac{\partial}{\partial q}\right), & a_{2}^{\dagger}=\frac{1}{\sqrt{2}}\left(q-\frac{\partial}{\partial q}\right)
\end{array}
$$

as

$$
\begin{aligned}
\mathcal{L}\left(a_{1}, a_{1}^{\dagger}, a_{2}, a_{2}^{\dagger}\right) & =\frac{i \omega}{2}\left(\left(a_{2}^{\dagger}+a_{2}\right)\left(a_{1}-a_{1}^{\dagger}\right)-\left(a_{1}^{\dagger}+a_{1}\right)\left(a_{2}-a_{2}^{\dagger}\right)\right) \\
& =i \omega\left(a_{2}^{\dagger} a_{1}-a_{1}^{\dagger} a_{2}\right)=\mathcal{L}_{N o r m}\left(a_{1}, a_{1}^{\dagger}, a_{2}, a_{2}^{\dagger}\right) .
\end{aligned}
$$

This operator is already in the Normal form and can be directly used in the functional integral for $\left\langle\bar{z}|\mathcal{U}(T, 0)| z^{\prime}\right\rangle$ :

$$
\begin{aligned}
& \left\langle\bar{z}_{1} \bar{z}_{2}|\mathcal{U}(T, 0)| z_{1}^{\prime} z_{2}^{\prime}\right\rangle=\int \mathcal{D} \psi_{1} \mathcal{D} \bar{\psi}_{1} \mathcal{D} \bar{\psi}_{2} \mathcal{D} \psi_{2} \\
& \exp \left\{\sum_{k=1}^{2} \bar{\psi}_{k}(T) \psi_{k}(T)-\frac{1}{2}\left|\bar{\psi}_{k}(T)\right|^{2}-\frac{1}{2}\left|\psi_{k}(0)\right|^{2}\right. \\
& \left.+\int_{0}^{T}\left(-\bar{\psi}_{1}(t) \dot{\psi}_{1}(t)-\bar{\psi}_{2}(t) \dot{\psi}_{2}(t)+\omega\left(\bar{\psi}_{2}(t) \psi_{1}(t)-\bar{\psi}_{1}(t) \psi_{2}(t)\right)\right) d t\right\}(14)
\end{aligned}
$$


where $\mathcal{D} \bar{\psi}_{1} \mathcal{D} \psi_{1}=\prod_{t} \frac{d \bar{\psi}_{1} d \psi_{1}}{2 \pi i}, \mathcal{D} \bar{\psi}_{2} \mathcal{D} \psi_{2}=\prod_{t} \frac{d \bar{\psi}_{2} d \psi_{2}}{2 \pi i}$ with the initial conditions:

$$
\begin{array}{cc}
\psi_{1}(0)=z_{1}^{\prime}, & \psi_{2}(0)=z_{2}^{\prime} \\
\bar{\psi}_{1}(T)=\overline{z_{1}}, & \bar{\psi}_{2}(T)=\overline{z_{2}}
\end{array}
$$

The functional integral is Gaussian and can be easily evaluated by finding the saddle point trajectory which are defined by the equations:

$$
\begin{array}{cc}
\dot{\psi}_{1}=-\omega \psi_{2}, & \dot{\bar{\psi}}_{1}=-\omega \bar{\psi}_{2}, \\
\dot{\psi}_{2}=\omega \psi_{1}, & \dot{\bar{\psi}}_{2}=\omega \bar{\psi}_{1} .
\end{array}
$$

Solutions of these equations are:

$$
\begin{aligned}
& \psi_{1}(t)=z_{2}^{\prime} \cos \omega t-z_{1}^{\prime} \sin \omega t, \\
& \psi_{2}(t)=z_{1}^{\prime} \cos \omega t+z_{2}^{\prime} \sin \omega t
\end{aligned}
$$

and

$$
\begin{aligned}
& \bar{\psi}_{1}(t)=\bar{z}_{2}^{\prime} \cos \omega(t-T)-\bar{z}_{1}^{\prime} \sin \omega(t-T), \\
& \bar{\psi}_{2}(t)=\bar{z}_{1}^{\prime} \cos \omega(t-T)+\bar{z}_{2}^{\prime} \sin \omega(t-T) .
\end{aligned}
$$

Therefore the integral turns into:

$$
\begin{aligned}
\left\langle\bar{z}_{1} \bar{z}_{2}|U(T, 0)| z_{1}^{\prime} z_{2}^{\prime}\right\rangle=\exp & \left\{\left(\bar{z}_{1} z_{1}^{\prime}+\bar{z}_{2} z_{2}^{\prime}\right) \cos \omega T-\frac{\left|z_{1}\right|^{2}}{2}-\frac{\left|z_{2}\right|^{2}}{2}\right. \\
& \left.-\left(\bar{z}_{1} z_{2}^{\prime}+\bar{z}_{2} z_{1}^{\prime}\right) \sin \omega T-\frac{\left|z_{1}^{\prime}\right|^{2}}{2}-\frac{\left|z_{2}^{\prime}\right|^{2}}{2}\right\}
\end{aligned}
$$

Combining together equations (6), (7), (8) and (15) one can get the following expression for the kernel of the evolution operator:

$$
\begin{array}{r}
U\left(p, q \mid p^{\prime}, q^{\prime}\right)=\int \frac{d z_{1} d \bar{z}_{1} d z_{2} d \bar{z}_{2} d z_{1}^{\prime} d \bar{z}_{1}^{\prime} d z_{2}^{\prime} d \bar{z}_{2}^{\prime}}{8 \pi^{5}} \\
\exp \left\{-\left|z_{1}\right|^{2}-\left|z_{2}\right|^{2}-\left|z_{1}^{\prime}\right|^{2}-\left|z_{2}^{\prime}\right|^{2}-\frac{z_{1}^{2}}{2}-\frac{z_{2}^{2}}{2}-\frac{z_{2}^{\prime 2}}{2}-\frac{z_{2}^{\prime 2}}{2}-\frac{p^{2}}{2}-\frac{q^{2}}{2}-\frac{p^{\prime 2}}{2}-\frac{q^{\prime 2}}{2}\right\} \\
\exp \left\{\sqrt{2}\left(z_{1} p+z_{2} q+\bar{z}_{1}^{\prime} p^{\prime}+\bar{z}_{2}^{\prime} q^{\prime}\right)+\left(\bar{z}_{1} z^{\prime}+\bar{z}_{2} z^{\prime}\right) \cos \omega T-\left(\bar{z}_{1}^{\prime} z_{2}^{\prime}+\bar{z}_{1}^{\prime} z_{2}^{\prime}\right) \sin \omega T\right\} .
\end{array}
$$

Now it is possible to show that this is Dirac $\delta$-function on the solutions of the classical mechanical equations of motion. Indeed, consider the following decomposition: 


$$
\begin{gathered}
\delta\left(x-x^{\prime}\right)=\left\langle x|I| x^{\prime}\right\rangle=\int\langle x \mid z\rangle\left\langle\bar{z} \mid z^{\prime}\right\rangle\left\langle\overline{z^{\prime}} \mid x^{\prime}\right\rangle \frac{d z d \bar{z} d z^{\prime} d \overline{z^{\prime}}}{4 i \pi i \pi} \\
=\int \frac{d z d \bar{z} d z^{\prime} d \overline{z^{\prime}}}{4 i \pi i \pi} \frac{1}{\sqrt{\pi}} \exp \left\{-\frac{|z|^{2}}{2}-\frac{x^{2}}{2}-\frac{z^{2}}{2}+\sqrt{2} x z\right\} \times \\
\exp \left\{-\frac{\left|\overline{z^{\prime}}\right|^{2}}{2}-\frac{x^{\prime 2}}{2}-\frac{{\overline{z^{\prime}}}^{2}}{2}+\sqrt{2} x^{\prime} \overline{z^{\prime}}-\frac{|z|^{2}}{2}-\frac{\left|z^{\prime}\right|^{2}}{2}-\bar{z} z^{\prime}\right\} .
\end{gathered}
$$

Using this decomposition and introducing a set of new variables:

$$
\begin{array}{ll}
\widetilde{z}_{1}^{\prime}=z_{1}^{\prime} \cos \omega T-z_{2}^{\prime} \sin \omega T, & \overline{\widetilde{z}}_{1}^{\prime}=\bar{z}_{1}^{\prime} \cos \omega T-\bar{z}_{2}^{\prime} \sin \omega T, \\
\widetilde{z}_{2}^{\prime}=z_{2}^{\prime} \cos \omega T+z_{1}^{\prime} \sin \omega T, & \overline{\widetilde{z}}_{1}^{\prime}=\bar{z}_{1}^{\prime} \cos \omega T+\bar{z}_{2}^{\prime} \sin \omega T,
\end{array}
$$

and

$$
\widetilde{p}^{\prime}=p^{\prime} \cos \omega T-q^{\prime} \sin \omega T, \quad \widetilde{q}^{\prime}=q^{\prime} \cos \omega T+p^{\prime} \sin \omega T .
$$

the kernel transforms into

$$
\begin{array}{r}
U\left(p, q \mid p^{\prime}, q^{\prime}\right)=\int \frac{d z_{1} d \bar{z}_{1} d z_{2} d \bar{z}_{2} d \widetilde{z}_{1}^{\prime} d \overline{\widetilde{z}}_{1}^{\prime} d \widetilde{z}_{2}^{\prime} d \overline{\widetilde{z}}_{2}^{\prime}}{\pi^{5}} \times \\
\exp \left\{-\left|z_{1}\right|^{2}-\left|z_{2}\right|^{2}-\left|\widetilde{z}_{1}\right|^{2}-\left|\widetilde{z}_{2}^{\prime}\right|^{2}\right\} \times \\
\exp \left\{-\frac{z_{1}^{2}}{2}-\frac{z_{2}^{2}}{2}-\frac{z_{2}^{\prime 2}}{2}-\frac{z_{2}^{\prime 2}}{2}-\frac{p^{2}}{2}-\frac{q^{2}}{2}-\frac{\widetilde{p}^{\prime 2}}{2}-\frac{\widetilde{q}^{2}}{2}\right\} \times \\
\exp \left\{\sqrt{2}\left(z_{1} p+z_{2} q+\overline{\widetilde{z}}_{1}^{\prime} \widetilde{p}^{\prime}+\overline{\widetilde{z}}_{2}^{\prime} \widetilde{q}^{\prime}\right)+\left(\bar{z}_{1} \widetilde{z}_{1}^{\prime}+\bar{z}_{2} \widetilde{z}_{2}^{\prime}\right)\right\} .
\end{array}
$$

which produces the required result

$$
U\left(p, q \mid p^{\prime}, q^{\prime}\right)=\delta\left(q-\widetilde{q}^{\prime}\right) \delta\left(p-\widetilde{p}^{\prime}\right) .
$$

This shows that the functional integral representation for the matrix elements of classical evolution operator indeed reproduces the Liouville flow on the classical phase space and is equivalent to the solution of classical Hamilton equations.

\section{New class of localization formulas for func- tional integrals}

In this section we describe a new class of functional integrals which can be evaluated exactly using their relationship to some classical dynamical systems. The main result is summarized in the following 
Theorem 1 For any function $V\left(q_{1} \ldots q_{n}\right)$ the following integral:

$$
\begin{array}{r}
I_{V}(z, \bar{z})=\int \mathcal{D} \psi_{1} \mathcal{D} \bar{\psi}_{1} \mathcal{D} \bar{\psi}_{2} \mathcal{D} \psi_{2} \exp \left\{\sum_{i=1}^{n} \sum_{k=1}^{2} \bar{\psi}_{k}^{i}(T) \psi_{k}^{i}(T)-\frac{1}{2}\left|\bar{\psi}_{k}^{i}(T)\right|^{2}-\frac{1}{2}\left|\psi_{k}^{i}(0)\right|^{2}\right\} \\
\exp \left\{\int_{0}^{T} \sum_{i=1}^{n}-\bar{\psi}_{1}^{i}(t) \dot{\psi}_{1}^{i}(t)-\bar{\psi}_{2}^{i}(t) \dot{\psi}_{2}^{i}(t)+\left(\bar{\psi}_{1}^{i}(t) \psi_{2}^{i}(t)-\bar{\psi}_{2}^{i}(t) \psi_{1}^{i}(t)\right)\right. \\
\left.+\left(\psi_{1}^{i}(t)-\bar{\psi}_{1}^{i}(t)\right) \mathcal{L}_{i, \text { norm }}^{(V)}(\psi, \bar{\psi})\right\}
\end{array}
$$

where $\mathcal{D} \bar{\psi}_{1} \mathcal{D} \psi_{1}=\prod_{t} \frac{d \bar{\psi}_{1} d \psi_{1}}{2 \pi i}, \mathcal{D} \bar{\psi}_{2} \mathcal{D} \psi_{2}=\prod_{t} \frac{d \bar{\psi}_{2} d \psi_{2}}{2 \pi i}$ with the initial conditions:

$$
\begin{gathered}
\psi_{1}(0)=z_{1}^{\prime}, \quad \psi_{2}(0)=z_{2}^{\prime} \\
\bar{\psi}_{1}(T)=\overline{z_{1}}, \quad \bar{\psi}_{2}(T)=\overline{z_{2}}
\end{gathered}
$$

and $\mathcal{L}_{i, n o r m}\left(a, a^{\dagger}\right)=\left.N\left(\frac{\partial V}{\partial q^{i}}-q^{i}\right)\right|_{q^{i}=\frac{1}{\sqrt{2}}\left(a_{2}^{i}+a_{2}^{\dagger i}\right)}$

is equal to

$$
\begin{aligned}
I_{V}(z, \bar{z})=\int \prod_{i=1}^{n} d p^{i} d q^{i}( & \frac{1}{\sqrt{\pi}} e^{-\frac{p^{i}(T)^{2}}{2}-\frac{q^{i}(T)^{2}}{2}-\frac{p^{\prime i 2}}{2}-\frac{q^{\prime i 2}}{2}} e^{-\frac{\left|z_{1}^{\prime i}\right|^{2}}{2}-\frac{\left|z_{2}^{\prime i}\right|^{2}}{2}-\frac{\left|\bar{z}_{1}^{i}\right|^{2}}{2}-\frac{\left|\bar{z}_{2}^{i}\right|^{2}}{2}} \\
& \left.e^{-\frac{z_{1}^{\prime i 2}}{2}-\frac{z_{2}^{\prime i 2}}{2}-\frac{\bar{z}_{1}^{i 2}}{2}-\frac{\bar{z}_{2}^{i 2}}{2}} e^{\sqrt{2}\left(z_{1}^{\prime i} p^{\prime i}+z_{2}^{\prime i} q^{\prime i}+\bar{z}_{1}^{i} p^{i}(T)+\bar{z}_{2} q^{i}(T)\right)}\right),
\end{aligned}
$$

where $\left\{p^{i}(T), q^{i}(T)\right\}$ are the solution of the classical Hamiltonian equations with the Hamilton function

$$
H=\frac{1}{2} \sum_{i=1}^{n} p^{i 2}+V\left(q^{1} \ldots q^{n}\right)
$$

for the time $t=T$ with initial conditions at $t=0$ being $\left\{p^{i}, q^{i}\right\}$.

Proof. Let us consider classical system with the following Hamilton function:

$$
H=\frac{1}{2} \sum_{i=1}^{n} p^{i 2}+V\left(q^{1} \ldots q^{n}\right)=\frac{1}{2} \sum_{i=1}^{n}\left(p^{i 2}+q^{i 2}\right)+V\left(q^{1} \ldots q^{n}\right)-\frac{1}{2} \sum_{i=1}^{n} q^{i 2} .
$$

The corresponding Liouville operator $\mathcal{L}$ will have the form:

$$
\mathcal{L}=i \sum_{i=1}^{n}\left(q^{i} \frac{\partial}{\partial p^{i}}-p^{i} \frac{\partial}{\partial q^{i}}\right)+i \sum_{i=1}^{n}\left(\frac{\partial V}{\partial q^{i}}-q^{i}\right) \frac{\partial}{\partial p^{i}} .
$$

Expressing this operator in terms of creation-annihilation operators $a_{1}^{i}, a_{2}^{i}, a_{1}^{\dagger i}, a_{2}^{\dagger i}$

$$
\begin{array}{ll}
a_{1}^{i}=\frac{1}{\sqrt{2}}\left(p^{i}+\frac{\partial}{\partial p^{i}}\right), & a_{1}^{i \dagger}=\frac{1}{\sqrt{2}}\left(p^{i}-\frac{\partial}{\partial p^{i}}\right) \\
a_{2}^{i}=\frac{1}{\sqrt{2}}\left(q^{i}+\frac{\partial}{\partial q^{i}}\right), & a_{2}^{i \dagger}=\frac{1}{\sqrt{2}}\left(q^{i}-\frac{\partial}{\partial q^{i}}\right)
\end{array}
$$


one can get

$$
\begin{aligned}
\mathcal{L}=i \sum_{i=1}^{n}\left(a_{2}^{\dagger i} a_{1}^{i}-a_{1}^{\dagger i} a_{2}^{i}\right) & +\left.\frac{i}{\sqrt{2}} \sum_{i=1}^{n} a_{1}^{i}\left(\frac{\partial V}{\partial q^{i}}-q^{i}\right)\right|_{q^{i}=\frac{1}{\sqrt{2}}\left(a_{2}^{i}+a_{2}^{\dagger i}\right)} \\
& -\left.\frac{i}{\sqrt{2}} \sum_{i=1}^{n} a_{1}^{\dagger i}\left(\frac{\partial V}{\partial q^{i}}-q^{i}\right)\right|_{q^{i}=\frac{1}{\sqrt{2}}\left(a_{2}^{i}+a_{2}^{\dagger i}\right)} .
\end{aligned}
$$

If the this equation is rewritten in the Normal form:

$$
\begin{aligned}
\mathcal{L}=i \sum_{i=1}^{n}\left(a_{2}^{\dagger i} a_{1}^{i}-a_{1}^{\dagger i} a_{2}^{i}\right) & +\frac{i}{\sqrt{2}} \sum_{i=1}^{n} \mathcal{L}_{i, n o r m}\left(a, a^{\dagger}\right) a_{1}^{i} \\
& -\frac{i}{\sqrt{2}} \sum_{i=1}^{n} a_{1}^{\dagger i} \mathcal{L}_{i, n o r m}\left(a, a^{\dagger}\right)
\end{aligned}
$$

where

$$
\mathcal{L}_{i, \text { norm }}\left(a, a^{\dagger}\right)=\left.N\left(\frac{\partial V}{\partial q^{i}}-q^{i}\right)\right|_{q^{i}=\frac{1}{\sqrt{2}}\left(a_{2}^{i}+a_{2}^{\dagger i}\right)}
$$

it is straightforward to put down the functional integral for the matrix elements of the evolution operator in the coherent state representation:

$$
\begin{array}{r}
\left\langle\bar{z}_{1} \bar{z}_{2}|\mathcal{U}(T, 0)| z_{1}^{\prime} z_{2}^{\prime}\right\rangle=\int \mathcal{D} \psi_{1} \mathcal{D} \bar{\psi}_{1} \mathcal{D} \bar{\psi}_{2} \mathcal{D} \psi_{2} \\
\exp \left\{\sum_{i=1}^{n} \sum_{k=1}^{2} \bar{\psi}_{k}^{i}(T) \psi_{k}^{i}(T)-\frac{1}{2}\left|\bar{\psi}_{k}^{i}(T)\right|^{2}-\frac{1}{2}\left|\psi_{k}^{i}(0)\right|^{2}\right\} \\
\exp \left\{\int_{0}^{T} \sum_{i=1}^{n}-\bar{\psi}_{1}^{i}(t) \dot{\psi}_{1}^{i}(t)-\bar{\psi}_{2}^{i}(t) \dot{\psi}_{2}^{i}(t)+\left(\bar{\psi}_{2}^{i}(t) \psi_{1}^{i}(t)-\bar{\psi}_{1}^{i}(t) \psi_{2}^{i}(t)\right)\right. \\
\left.-\left(\psi_{1}^{i}(t)-\bar{\psi}_{1}^{i}(t)\right) \mathcal{L}_{i, \text { norm }}(\psi, \bar{\psi}) d t\right\}
\end{array}
$$

where $\mathcal{D} \bar{\psi}_{1} \mathcal{D} \psi_{1}=\prod_{t} \prod_{l=1}^{n} \frac{d \bar{\psi}_{1}^{l} d \psi_{1}^{l}}{2 \pi i}, \mathcal{D} \bar{\psi}_{2} \mathcal{D} \psi_{2}=\prod_{t} \prod_{l=1}^{n} \frac{d \bar{\psi}_{2}^{l} d \psi_{2}^{l}}{2 \pi i}$ with the initial conditions:

$$
\begin{aligned}
& \psi_{1}(0)=z_{1}^{\prime}, \quad \psi_{2}(0)=z_{2}^{\prime} \\
& \bar{\psi}_{1}(T)=\overline{z_{1}}, \quad \bar{\psi}_{2}(T)=\overline{z_{2}}
\end{aligned}
$$

The same matrix element can be found as

$$
\begin{array}{r}
U\left(\left\{\bar{z}_{1}^{i}, \bar{z}_{2}^{i}\right\} \mid\left\{z_{1}^{\prime i}, z_{2}^{\prime i}\right\}\right)=\left\langle\left\{\bar{z}_{1}^{i}, \bar{z}_{2}^{i}\right\}|\mathcal{U}(0, T)|\left\{z_{1}^{\prime i}, z_{2}^{\prime i}\right\}\right\rangle \\
=\int \prod_{i=1}^{n} d p^{i} d p^{i} d q^{i} d q^{i}\left\langle\left\{\bar{z}_{1}^{i}, \bar{z}_{2}^{i}\right\} \mid\left\{p^{i}, q^{i}\right\}\right\rangle\left\langle\left\{p^{\prime}, q^{\prime i}\right\} \mid\left\{z_{1}^{\prime i}, z_{2}^{\prime i}\right\}\right\rangle \times \\
\left\langle\left\{p^{i}, q^{i}\right\}|\mathcal{U}(0, T)|\left\{p^{\prime i}, q^{\prime i}\right\}\right\rangle .
\end{array}
$$


Using equations (6) and (7) one can easily get:

$$
\begin{array}{r}
U\left(\left\{\bar{z}_{1}^{i}, \bar{z}_{2}^{i}\right\} \mid\left\{z_{1}^{\prime i}, z_{2}^{\prime i}\right\}\right) \\
=\int \prod_{i=1}^{n} d p^{i} d p^{\prime i} d q^{i} d q^{\prime i}\left\langle\left\{p^{i}, q^{i}\right\}|\mathcal{U}(0, T)|\left\{p^{\prime i}, q^{\prime i}\right\}\right\rangle \times \\
\mathcal{R}\left(\left\{p^{i}, q^{i}\right\},\left\{p^{\prime i}, q^{\prime i}\right\},\left\{z_{1}^{\prime i}, z_{2}^{\prime i}\right\},\left\{\bar{z}_{1}^{i}, \bar{z}_{2}^{i}\right\}\right),
\end{array}
$$

where

$$
\begin{aligned}
& \mathcal{R}\left(\left\{p^{i}, q^{i}\right\},\left\{p^{\prime i}, q^{\prime i}\right\},\left\{z_{1}^{\prime i}, z_{2}^{\prime i}\right\},\left\{\bar{z}_{1}^{i}, \bar{z}_{2}^{i}\right\}\right) \\
& =\left\langle\left\{\bar{z}_{1}^{i}, \bar{z}_{2}^{i}\right\} \mid\left\{p^{i}, q^{i}\right\}\right\rangle\left\langle\left\{p^{\prime i}, q^{\prime i}\right\} \mid\left\{z_{1}^{i}, z_{2}^{i i}\right\}\right\rangle \\
& =\prod_{i=1}^{n}\left(\frac{1}{\sqrt{\pi}} e^{-\frac{p^{i 2}}{2}-\frac{q^{i 2}}{2}-\frac{p^{\prime i 2}}{2}-\frac{q^{\prime i 2}}{2}} e^{-\frac{\left|z_{1}^{\prime i}\right|^{2}}{2}-\frac{\left|z_{2}^{\prime i}\right|^{2}}{2}-\frac{\left|\bar{z}_{1}^{i}\right|^{2}}{2}-\frac{\left|\bar{z}_{2}^{i}\right|^{2}}{2} \times}\right. \\
& \left.e^{-\frac{z_{1}^{\prime 2}}{2}-\frac{z_{2}^{\prime 2}}{2}-\frac{\bar{z}_{1}^{i 2}}{2}-\frac{\bar{z}_{2}^{i 2}}{2}} e^{\sqrt{2}\left(z_{1}^{\prime i} p^{\prime i}+z_{2}^{\prime i} q^{\prime i}+\bar{z}_{1}^{i} p^{i}+\bar{z}_{2}^{i} q^{i}\right)}\right) .
\end{aligned}
$$

Taking into account that the classical evolution operator is a delta-function on the solution of the classical equations it is easy to see that expression (25) can be re-arranged as:

$$
\begin{array}{r}
U\left(\left\{\bar{z}_{1}^{i}, \bar{z}_{2}^{i}\right\} \mid\left\{z_{1}^{\prime i}, z_{2}^{i}\right\}\right)=\int \prod_{i=1}^{n} d p^{\prime i} d q^{i i}\left(\frac{1}{\sqrt{\pi}} e^{-\frac{p^{i}(T)^{2}}{2}-\frac{q^{i}(T)^{2}}{2}-\frac{p^{\prime i 2}}{2}-\frac{q^{\prime i 2}}{2}}\right. \\
\left.e^{-\frac{\left|z_{1}^{\prime i}\right|^{2}}{2}-\frac{\left|z_{2}^{\prime i}\right|^{2}}{2}-\frac{\left|\bar{z}_{1}^{i}\right|^{2}}{2}-\frac{\left|\bar{z}_{\mid}^{i}\right|^{2}}{2}} e^{-\frac{z_{1}^{\prime i 2}}{2}-\frac{z_{2}^{\prime i 2}}{2}-\frac{\bar{z}_{1}^{i 2}}{2}-\frac{\bar{z}_{2}^{i 2}}{2}} e^{\sqrt{2}\left(z_{1}^{\prime i} p^{\prime i}+z_{2}^{\prime i} q^{\prime i}+\bar{z}_{1}^{i} p^{i}(T)+\bar{z}_{2} q^{i}(T)\right)}\right) .
\end{array}
$$

where $\left\{p^{i}(T), q^{i}(T)\right\}$ are the solution of the classical Hamiltonian equations for the time $t=T$ with initial conditions at $t=0$ being $\left\{p^{\prime i}, q^{i}\right\}$. This gives the formula for exact evaluation of the described class of functional integrals in terms of solutions of the corresponding classical equations of motion. In the case of integrable systems when the solutions can be written down explicitly, the functional integrals will be evaluated explicitly as well. This completes the proof.

\section{Concluding Remarks}

In this paper we suggested a new functional integral representation for classical evolution operator. Having calculated this expression one can study time evolution of any classical observable as well as statistical properties of classical systems. As a by-product we also found a set of quantum mechanical systems 
which are related to the classical mechanics and can be solved exactly by reducing the problem to solutions of the corresponding classical equations.

As we pointed in the Introduction the existence of the functional integral representation opens interesting technical opportunities. Let us list here the main directions which, we believe, promise interesting results in future:

1. Study of classical dynamical systems in the presence of random forces. Since the evolution operator can be formally written for any interaction structure, one can derive directly a functional integral representation for averaged dynamics of classical observables as well as their statistical characteristics in close analogy with the corresponding quantum case 4]. One of applications in this case would be study of classical chaotic systems by means of non-perturbative field-theoretical methods.

2. Collective variables in classical dynamical systems. One can apply functional form of Stratonovich-Hubbard transformation to introduce collective variables into the classical systems with many interacting degrees of freedom. This might be an interesting bridge between classical n-particle dynamics and classical statistical physics.

3. Investigation of possibility to introduce dissipative forces into the formalism. This can potentially lead to a new instrument of study nonequilibrium open systems and self-organization.

4. Exact solutions of quantum mechanical or field theoretical problems in terms of the related classical trajectories. One can see the relation established here between as certain class of quantum systems and some classical systems as a form of localization formulas for the corresponding quantum functional integrals. It is interesting to see how wide is the class and if the formalism can be generalized to include even more quantum systems (not necessary 2n-dimensional ones).

We hope to return to these issues in our future publications.

Acknowledgement We would like to thank good old Sasha Stepanenko for discussion of the subject in 1992. We also grateful to Serge Levin for questions and comments.

\section{References}

[1] L.D.Faddeev, V.N.Popov, Phys. Lett. B 25 (1978) 
[2] 't.Hooft G., Phys. Rev. Lett. 37:8 (1976)

[3] R.Rajaraman, "Solitons and Instantons", Noth-Holland Publishing Co. (1982)

[4] K.B.Efetov, "Supersymmetry in Disorder and Chaos", Cambridge University Press, New York. (1997)

[5] V.N.Popov, "Functional integrals and collective exitations", Cambrige University Press. (1987)

[6] E. Witten, Quantum Field Theory And The Jones Polynomial, Comm. Math. Phys. 121 (1989) 351.

[7] Avarez-Gaume, L: Supersymmetry and the Atiyah-Singer Index Theorem,Commun.Math.Phys 90, 161-173 (1983)

[8] N.V.Borisov, K.N.Ilinski, N=2 Supersymmetric Quantum Mechanics onRiemann surfaces with meromorphic superpotentials, Commun.Math.Phys 161, 177-194 (1994); N.V.Borisov, K.N.Ilinski, G.V.Kalinin, New index formulas as a meromorphic generalization of Chern-Gauss-Bonnet theorem,Lett.Math.Phys. 20, 21-27, (1997)

[9] E.Gozzi, M.Reuter,W.D.Thacker, Phys. Rev.D 40 (1989) 3363; A.A.Abrikosov (Jr.), Nucl. Phys. B 382 (1992) 581; E.Deotto and E.Gozzi and D.Mauro, Hilbert Space Structure in Classical Mechanics: (I), Rend. Sem. Mat. Univ. Politec. Torino 54, 269-277 (1996); E.Gozzi and M.Regini, Addenda and corrections to work done on the path-integral approach to classical mechanics, Phys. Rev. D 62067702 (2000); Hilbert Space Structure in Classical Mechanics: (II), J. Math. Phys. 44 5937-5957 (2003);E.Gozzi, Functional Techniques in Classical Mechanics, Nucl. Phys. Proc. Suppl. 104 (2001); A.A.Abrikosov(jr) and E.Gozzi and D.Mauro, Time and Geometric Quantization, Mod. Phys. Lett. A18, 2347-2354 (2003) 